Provided for non-commercial research and education use. Not for reproduction, distribution or commercial use.

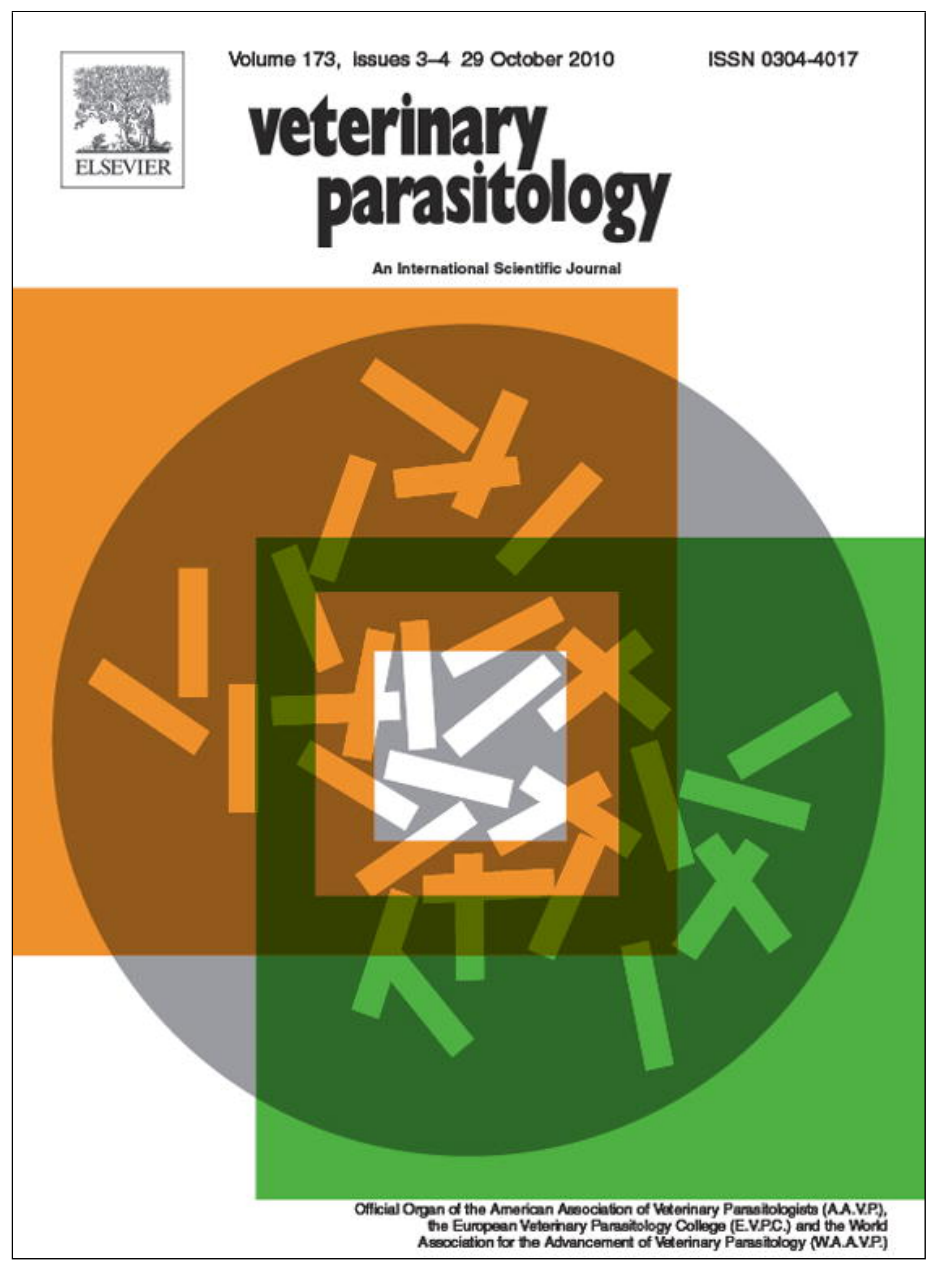

This article appeared in a journal published by Elsevier. The attached copy is furnished to the author for internal non-commercial research and education use, including for instruction at the authors institution and sharing with colleagues.

Other uses, including reproduction and distribution, or selling or licensing copies, or posting to personal, institutional or third party websites are prohibited.

In most cases authors are permitted to post their version of the article (e.g. in Word or Tex form) to their personal website or institutional repository. Authors requiring further information regarding Elsevier's archiving and manuscript policies are encouraged to visit:

http://www.elsevier.com/copyright 


\title{
Effect of Eimeria acervulina infection history on the immune response and transmission in broilers
}

\author{
F.C. Velkers ${ }^{\mathrm{a}, \mathrm{b}, *, 1}$, W.J.C. Swinkels ${ }^{\mathrm{a}, 1}{ }$, J.M.J. Rebel ${ }^{\mathrm{c}}$, A. Bouma ${ }^{\mathrm{a}}$, A.J.J.M. Daemen ${ }^{\mathrm{a}}$, \\ D. Klinkenberg ${ }^{\mathrm{a}}$, W.J.A. Boersma ${ }^{\mathrm{c}}$, J.A. Stegeman ${ }^{\mathrm{a}}$, M.C.M. de Jong ${ }^{\mathrm{b}}$, J.A.P. Heesterbeek ${ }^{\mathrm{a}}$ \\ a Department of Farm Animal Health, Faculty of Veterinary Medicine, Utrecht University, P.O. Box 80151, 3508 TD Utrecht, The Netherlands \\ b Quantitative Veterinary Epidemiology Group, Wageningen Institute of Animal Sciences, Wageningen University, P.O. Box 338, 6700 AH Wageningen, The \\ Netherlands \\ c Central Veterinary Institute, P.O. Box 65, 8200 AB Lelystad, The Netherlands
}

\section{A R T I C L E I N F O}

\section{Article history:}

Received 4 December 2009

Received in revised form 8 July 2010

Accepted 15 July 2010

\section{Keywords:}

Eimeria acervulina

Broilers

Transmission

Immunity

Infection dynamics

Immuno-epidemiology

\begin{abstract}
A B S T R A C T
Heterogeneity in exposure to Eimeria spp. of chickens in a flock will result in differences between individual birds in oocyst output and acquired immunity, which subsequently affects transmission of the parasite in the population. The aim of this study was to quantify effects of previous infection of broilers with Eimeria acervulina on immune responses, oocyst output and transmission. A transmission experiment was carried out with pair-wise housed broilers, that differed in infection history. This "infection history" was achieved by establishment of a primary infection by inoculation of birds with 50,000 sporulated $E$. acervulina oocysts at day 6 of age ("primed"); the other birds did not receive a primary infection ("naïve"). The actual transmission experiment started at day 24 of age: one bird (I) was inoculated with 50,000 sporulated oocysts and was housed together with a noninoculated contact bird $(C)$. Oocyst excretion and parameters describing transmission, i.e. the number of infected $C$ birds and time passed before start of excretion of $C$ birds, were determined from day 28 to day 50 for six pairs of four different combinations of $I$ and $C$ birds $(I-C)$ : naïve-naïve, naïve-primed, primed-naïve and primed-primed. Immune parameters, $\mathrm{CD}^{+}, \mathrm{CD}^{+}, \alpha \beta \mathrm{TCR}^{+}$and $\gamma \delta \mathrm{TCR}^{+} \mathrm{T}$ cells and macrophages in duodenum, were determined in an additional 25 non-primed, non-inoculated control birds, and in the naïve-naïve and naïve-primed groups, each group consisting of 25 pairs. Although the numbers of $\mathrm{CD}^{+}$ T cells and $\gamma \delta \mathrm{TCR}^{+} \mathrm{T}$ cells increased after primary infection, none of the immunological cell types provided an indication of differences in infectivity, susceptibility or transmission between birds. Oocyst output was significantly reduced in primed $I$ and $C$ birds. Transmission was reduced most in the primed-primed group, but nonetheless transmission occurred in all groups. This study also showed that acquired immunity significantly reduced oocyst output after inoculation and contact-infection, but not sufficiently to prevent transmission to contact-exposed birds.
\end{abstract}

(c) 2010 Elsevier B.V. All rights reserved.

\footnotetext{
* Corresponding author at: Department of Farm Animal Health, Faculty of Veterinary Medicine, Utrecht University, P.O. Box 80151, 3508 TD Utrecht, The Netherlands. Tel.: +31 30 2534447; fax: +31 302521887. E-mail address: f.c.velkers@uu.nl (F.C. Velkers).

1 These two authors contributed equally to this work.
}

\section{Introduction}

One of the most prevalent infections in commercial poultry is coccidiosis, caused by various species of the genus Eimeria. Infection with Eimeria spp. may cause enteritis, mortality, welfare problems and economic losses due to production losses and costs for treatment or 
prevention (Shirley et al., 2005). The majority of the current control strategies rely on the use of anticoccidials, supplemented with live attenuated vaccines. Increasing resistance among Eimeria species (Chapman, 1997; Peek and Landman, 2003), legislative restrictions against infeed medication, and the lack of success in producing cost-effective vaccines, stimulate the demand for alternative approaches of the problem. More research, aimed at increasing insight in processes that result in a protective immune response and towards providing adequate indicators of protection against infection, might contribute to the development of alternative intervention strategies.

Immune responses, developed after (repeated) infection with parasites such as Eimeria spp. affect the susceptibility and infectivity of individual birds (Rose, 1987; Lillehoj, 1988; Stiff and Bafundo, 1993; Williams, 1995; Claerebout and Vercruysse, 2000). As a result of differences in the exposure of birds to infectious oocysts from the environment, birds in a group are likely to differ in their infection history, which will result in a heterogeneous population with respect to infectivity and immunity. This heterogeneity will cause variability between birds with respect to oocyst excretion and their response after subsequent ingestion of oocysts in the population, which implies that host-pathogen interactions in individuals will affect the transmission of the parasite between individuals in a population and vice versa (Severins et al., 2007).

Most studies carried out in the last decades focused either on immunity (Lillehoj, 1988; Stiff and Bafundo, 1993; Williams, 1995) or epidemiology (Graat et al., 1998; Williams, 1998), but it is the mutual interaction between host and pathogen ("within-host dynamics"), and between infectious and susceptible hosts in a flock and the environment ("between-host dynamics") that determines the dynamics of the infection with the pathogen in a flock. Although this seems straightforward, the outcome of these interactions may give rise to non-linear effects, which may result in a counterintuitive and an unpredictable course of the infection (Roberts and Heesterbeek, 1995, 1998; Graat et al., 1996; Klinkenberg and Heesterbeek, 2007). Therefore, to provide more insight in the course of the disease coccidiosis in a flock, the dynamics of the immune reaction in individual hosts and transmission of the infection between hosts should be studied together.

The aim of the current study was to examine the relation between immune responses and transmission between birds. A transmission experiment was carried out with broilers with different (artificially induced) infection histories to Eimeria acervulina, presumed to result in different levels of acquired immunity. Oocyst output kinetics, intestinal immune responses in individual birds and transmission characteristics were quantified to determine whether immune parameters correlated with infectivity or susceptibility of birds, which would be useful to characterize differences between individual hosts, and to monitor and predict transmission of infection between birds. The approach described in this paper might also be applied to other (parasitic or protozoan) diseases.

\section{Materials and methods}

\subsection{Chickens and management}

At day 0 of age (day 0 of the experiment), 173 male SPF broiler chickens were obtained from the Animal Health Service (AHS, Deventer, The Netherlands). The chicks originated from a specified pathogen free (SPF) parent flock, a crossbred of Cobb, Hybro and Ross, kept in an Eimeria spp. free environment. Until day 25 of age birds were housed in groups in battery cages (Tecniplast ${ }^{\circledR}$, Tecnilab-BMI, The Netherlands). From day 25 onwards, chicks were housed in pairs on wood shavings in randomly allocated floor pens (height $\times$ width $\times$ depth $=80 \mathrm{~cm} \times 60 \mathrm{~cm} \times 50 \mathrm{~cm}$ ). Control birds were housed in groups of five birds in battery cages on wood shavings.

Room temperature was $32^{\circ} \mathrm{C}$ at day 0 and was gradually decreased to $18^{\circ} \mathrm{C}$ at day 50 . The chicks were subjected to a lighting scheme of $23 \mathrm{~h}$ of light per day. A broiler ration (12 MJ/kg metabolizable energy) without anticoccidial drugs, and drinking water were available ad libitum.

The birds were observed twice daily for signs of illness or welfare impairment. Birds were housed, handled and treated following approval by the Animal Experiments Committee of Utrecht University (Utrecht, The Netherlands), in accordance with the Dutch Experiments on Animals Act.

\subsection{Experimental design}

An experiment was carried out with pairs of birds consisting of one inoculated $(I)$ and one non-inoculated contact (C) bird, referred to as a pair-wise transmission experiment (Velthuis et al., 2007).

The transmission experiment started when the birds were 24 days old with inoculation of one bird per pair (I). Seventy-four I birds were orally inoculated at day 24 with 50,000 sporulated E. acervulina oocysts (Weybridge W119, supplied by AHS; originally provided by the Central Veterinary Laboratory in Weybridge, United Kingdom), suspended in $1 \mathrm{ml}$ of tap water. The 74 contact birds $(C)$ were not inoculated. $I$ and $C$ birds were housed pairwise in a floor pen from day 25 onwards until the end of the experiment. Transmission of the infection was subsequently measured by determining for each pair if and when the contact bird became infected, based on oocyst excretion.

At the start of the transmission trial, birds differed in infection history, resulting in different immune status. The infection history was induced by artificial inoculation of 49 birds at day 6 , with a single oral dose of 50,000 sporulated E. acervulina oocysts. At that age, these birds received a primary infection, denoted by " $P$ " and referred to as "primed". The other birds did not receive such a primary infection (denoted by " $N$ " and referred to as "non-primed" or "naïve"). From day 6 to day 24 of age, birds treated equally were housed together in battery cages.

The transmission experiment was carried out with pairs of birds in four different combinations: 
Table 1

Experimental design: composition and notation of groups, inoculation and measurements.

\begin{tabular}{|c|c|c|c|c|c|c|}
\hline Group & Bird & $\begin{array}{l}\text { Inoculation day } 6 \\
\text { (primary infection) }^{\mathrm{a}}\end{array}$ & Status at day $24^{\mathrm{a}}$ & Inoculation day $24^{\mathrm{b}}$ & $\#$ of chicks ${ }^{c}$ & Parameters tested $^{\mathrm{c}}$ \\
\hline Control & Control & None & Naïve & Non-inoculated & 25 & Immune parameters \\
\hline $1: N N$ & $I_{N N}$ & None & Naïve & Inoculated & $\begin{array}{r}6 \\
25\end{array}$ & $\begin{array}{l}\text { Transmission/oocyst output } \\
\text { Immune parameters }\end{array}$ \\
\hline $1: N N$ & $C_{N N}$ & None & Naïve & Non-inoculated & $\begin{array}{r}6 \\
25\end{array}$ & $\begin{array}{l}\text { Transmission/oocyst output } \\
\text { Immune parameters }\end{array}$ \\
\hline 2: NP & $I_{N P}$ & None & Naïve & Inoculated & $\begin{array}{r}6 \\
25\end{array}$ & $\begin{array}{l}\text { Transmission/oocyst output } \\
\text { Immune parameters }\end{array}$ \\
\hline $2: N P$ & $C_{N P}$ & Inoculated & Primed & Non-inoculated & $\begin{array}{r}6 \\
25\end{array}$ & $\begin{array}{l}\text { Transmission/oocyst output } \\
\text { Immune parameters }\end{array}$ \\
\hline 3: $P N$ & $I_{P N}$ & Inoculated & Primed & Inoculated & 6 & Transmission/oocyst output \\
\hline $3: P N$ & $C_{P N}$ & None & Naïve & Non-inoculated & 6 & Transmission/oocyst output \\
\hline 4: $P P$ & $I_{P P}$ & Inoculated & Primed & Inoculated & 6 & Transmission/oocyst output \\
\hline $4: P P$ & $C_{P P}$ & Inoculated & Primed & Non-inoculated & 6 & Transmission/oocyst output \\
\hline
\end{tabular}

a Before start of the transmission experiment at day 24, the birds were either primed/had received a primary infection (i.e. were inoculated at day 6 with 50,000 sporulated E. acervulina oocysts) to artificially induce infection history, or were naïve/non-primed (i.e. were not inoculated at day 6).

b At day 24 birds were either inoculated ( $I$ birds) or not ( $C$ birds and negative control birds) to start the infectious process in the pair-wise transmission experiment.

c Oocyst output and transmission were determined for $I$ and $C$ birds for six pairs of Groups 1-4 from days 28-50. Immune parameters were determined in 25 control birds and an additional 25 pairs of $I$ en $C$ birds from Groups $1(N N)$ and $2(N P)$ at days 28, 31, 33, 36 and 39. Five birds of each group were used per day of testing.

- Group 1, "NN", Naïve inoculated bird and naïve contact bird ( $I_{N N}$ and $C_{N N}$ bird).

- Group 2, "NP", Naïve inoculated bird and primed contact bird $\left(I_{N P}\right.$ and $C_{N P}$ bird).

- Group 3, "PN", Primed inoculated bird and naïve contact bird ( $I_{P N}$ and $C_{P N}$ bird).

- Group 4, "PP", Primed inoculated and primed contact bird ( $I_{P P}$ and $C_{P P}$ bird).

Note that for the group notation the abbreviation for the infection status of the inoculated bird, " $N$ " or " $P$ ", precedes that of the contact bird. For individual birds, the notation starts with the status in the transmission experiment, i.e. either inoculated, " $I$ " bird or non-inoculated contact, " $C$ " bird, followed by a subscript to denote to which group they belong, i.e. $N N, N P, P N$ or $P P$.

Transmission and oocyst excretion were quantified for all four groups, consisting of six pairs each. Immune parameters were quantified, based on a study by Cornelissen et al. (2009), for Groups 1 and 2 ( $N N$ and $N P$, respectively), using 25 additional pairs each; five birds were sacrificed for quantification of immune parameters at each of the days $28,31,33,36$ and 39 . For this immunological study we also included another five groups of five non-inoculated, nonprimed chickens that served as "negative controls". Group composition and treatments are explained in Table 1 and illustrated with a flow chart in Fig. 1.

\subsection{Immune parameters}

Five pairs of the $N N$ and $N P$ group and five birds of the control group were killed by cervical dislocation at days 28, 31, 33, 36 and 39 (Table 1). One $\mathrm{cm}$ of the duodenal loop was snap-frozen in liquid nitrogen and stored at $-70^{\circ} \mathrm{C}$ until further examination by immunohistochemistry. Immunohistological staining by an indirect immunoperoxidase method was performed on frozen duodenum sections ( $8 \mu \mathrm{m}$ thick), collected from chickens of $28,31,33,36$ and 39 days of age as described by
Cornelissen et al. (2009). In short, endogenous peroxidase was inhibited and were subsequently incubated for $1 \mathrm{~h}$ with monoclonal antibodies against $\mathrm{CD} 4^{+} \mathrm{T}$ cells (1:200; CT-4, Southern Biotech), CD8 ${ }^{+}$T cells (1:200; CT8 , Southern Biotech), $\alpha \beta \mathrm{TCR}^{+} \mathrm{T}$ cells (1:50; TCR2, Southern Biotech), $\gamma \delta \mathrm{TCR}^{+} \mathrm{T}$ cells (1:400; TCR1, Southern Biotech) or monocytes/macrophages (1:50; KUL-01, Southern Biotech) followed by peroxidase-conjugated rabbit anti-mouse Ig (1:80; P0161, Dakopatts, Denmark). Peroxidase activity was detected by $0.05 \%$ 3,3-diaminobenzidine (DAB) in $0.1 \mathrm{M}$ Tris $-\mathrm{HCl}$ solution ( $\mathrm{pH} 7.5$ ) containing $0.03 \% \mathrm{H}_{2} \mathrm{O}_{2}$. The images were analyzed with Image-Pro Plus (version 5.1 , media cybernetics) to quantify the number of stained T cells per $0.5 \mathrm{~mm}^{2}$. Per bird five images were acquired and analyzed and the mean of the five $\log _{10}$-transformed $T$ cell counts per bird was used for the statistical analysis.

Immunological data was analyzed per group to determine whether elevated or decreased levels of the different cell types could be identified as indicators of infection or acquired/protective immunity. Therefore, a linear mixed model was applied using SAS for Windows 9.1 (SAS Institute Inc., Cary, NC, USA) with $\log _{10}$-transformed mean cell counts as dependent variable and litter cage as random factor. Bird type (five different bird types, i.e. $I_{N N}, C_{N N}$, $I_{N P}, C_{N P}$ and control birds), day (days 28, 31, 33, 36 and 39) and the interaction group*day were entered into the model as explanatory variables. The two-tailed partial $F$ test (type III) was used as the elimination criterion for the model building and the fit of the model was assessed by the Akaike's Information Criterion. The best model for $\mathrm{CD}^{+}$and $\mathrm{CD}^{+} \mathrm{T}$ cells consisted of day and the interaction between group*day. The final model for $\alpha \beta \mathrm{TCR}^{+}$ and $\gamma \delta \mathrm{TCR}^{+}$cells contained day, group and group*day as explanatory variables. Differences in $\mathrm{T}$ cell counts were assessed per day between $I$ birds of both groups and control birds, between $C$ birds of both groups and control birds, between $I_{N N}$ and $I_{N P}$ birds and between $C_{N N}$ and $C_{N P}$ birds. Bonferroni corrected pair-wise multiple $t$-tests were carried out to test the difference between each above men- 


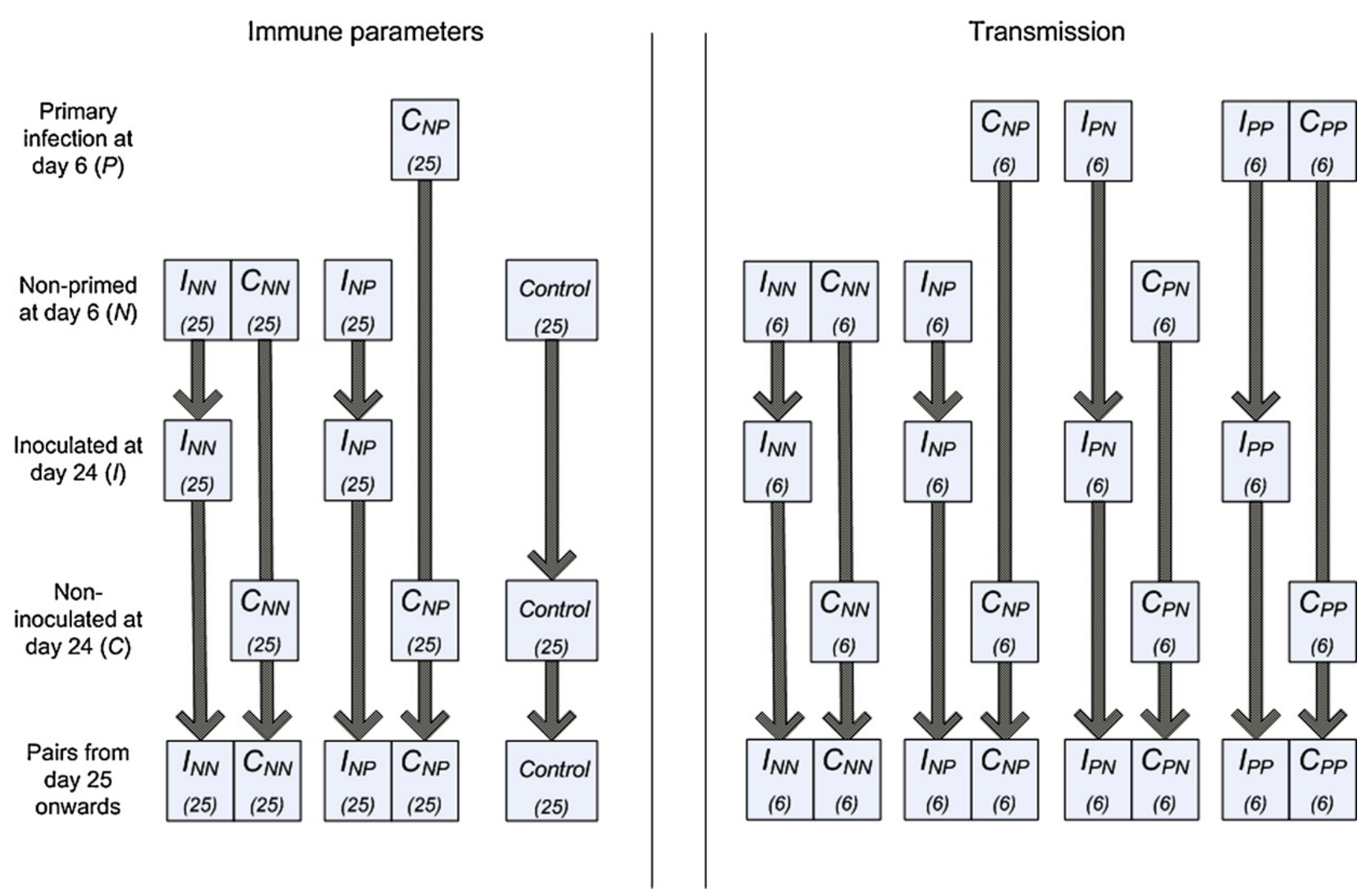

Fig. 1. Flow chart of the study design, illustrating the treatments at different times during the experiment. At day 6 , birds either received a primary infection $(P)$ or not $(N)$ to induce different infection histories and were inoculated $(I)$ or not $(C)$ at day 24 . From day 25 onwards, four different combinations of $I$ and $C$ birds were housed pair-wise in floor pens. Immune parameters (left side of the flow chart) were determined for two groups and transmission parameters (right) for four different groups. The notations for the different bird types in the experiment $\left(I_{N N}, C_{N N}, I_{N P}, C_{N P}, I_{P N}, C_{P N}, I_{P P}, C_{P P}\right.$ and control birds) are given with the number of birds between brackets.

tioned pair of means. Model assumptions were evaluated by examining normality and equality of variances of the residuals. The level of statistical significance was set at $P<0.05$.

\subsection{Droppings collection, processing and oocyst counts}

The number of oocysts per gram of faeces (OPG) was quantified using data from six pairs of birds from Groups 1 to 4 . Single individual droppings were collected daily from days 10 to 23 from primed birds and daily from days 28 to 50 from all $I$ and $C$ birds, or until no oocysts were detected for three consecutive days. The single droppings were collected and analyzed according to a procedure described by Velkers et al. (2010). Briefly, each chick was placed in a cardboard box with a clean paper sheet for 1-2 h. The single dropping was weighed and OPG was determined, according to a modification of a McMaster oocyst counting chamber technique described by Long and Rowell (1958).

The detection limit of the McMaster technique was 83 oocysts per $\mathrm{g}$ of faeces for a faecal sample of $4 \mathrm{~g}$. If no oocysts were found, a modification of the sedimentation-flotation (SF) technique was applied (Long et al., 1976), which is a more sensitive technique, though only qualitative (Mes, 2003).

For the oocyst excretion patterns, the average oocyst production was determined by calculating the mean of the $\log _{10}$-transformed OPG per day for $I$ and $C$ birds per group. As a measure for the total number of excreted oocysts, the area under the curve (AUC) of oocyst output was calculated for each bird from the daily non-transformed OPG results. AUC was $\log _{10}$-transformed, to obtain normally distributed data, and the mean $\log _{10}$-transformed AUC $\left(\log _{10}\right.$ AUC) was calculated for $I$ and $C$ birds per group. Also the period in which OPG was intermittently above zero (last day of positive OPG minus first day of positive OPG results = PERIOD) and the highest measured $\log _{10}$ OPG peak output (PEAK) were determined.

These data were analyzed per group to determine how infection history influences oocyst excretion characteristics. Therefore, a linear mixed model was applied with $\log _{10}$ AUC, $\sqrt{ }$ PERIOD or PEAK as dependent variable, bird type (eight different bird types, i.e. $I$ and $C$ birds of each of the four different groups) as fixed factor and litter cage as random factor using SAS for Windows 9.1 (SAS Institute Inc., Cary, NC, USA). The two-tailed partial F-test (type III) was used as the elimination criterion for the model building and the fit of the model was assessed by the Akaike's Information Criterion. Bonferroni corrected pair-wise multiple $t$-tests were carried out to test the difference between each pair of means. Model assumptions were evaluated by examining normality and equality of variances of the residuals. The level of statistical significance was set at $P<0.05$. 

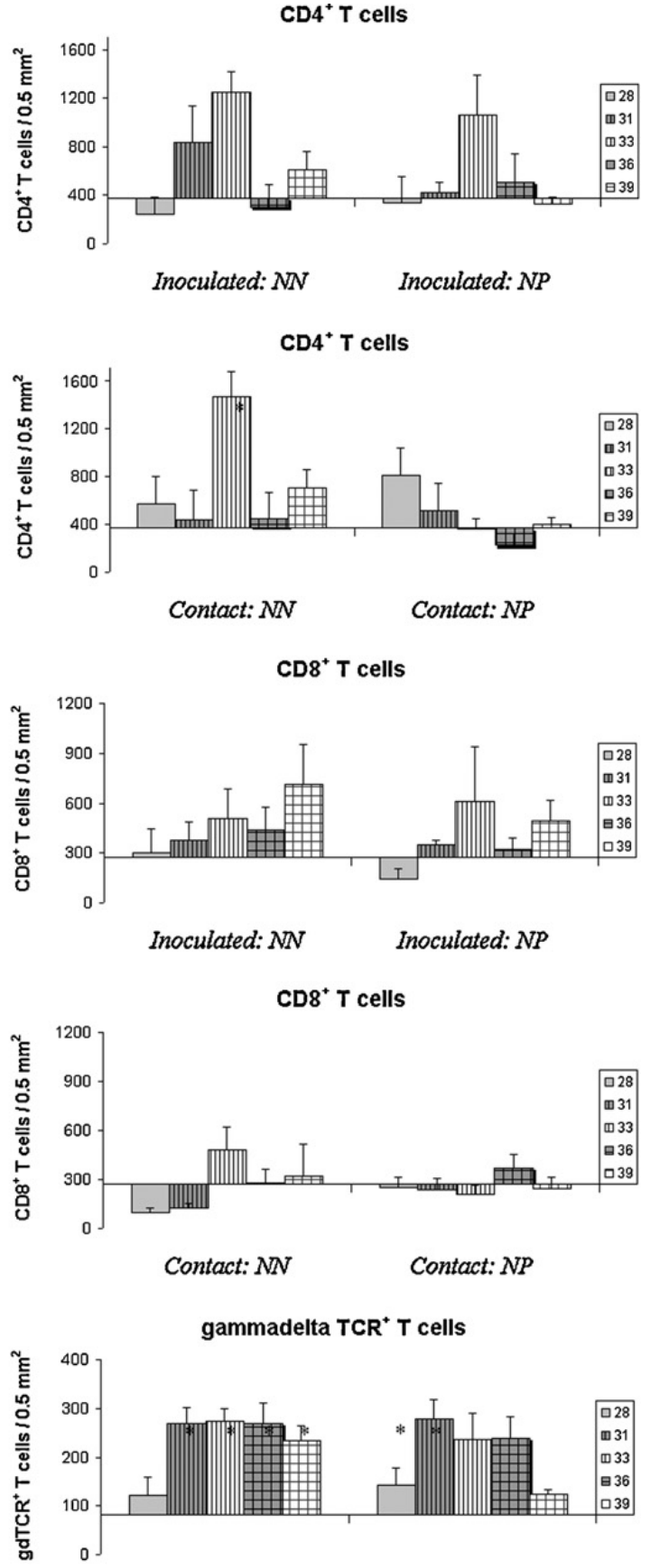

Inoculated: $N N \quad$ Inoculated: $N P$

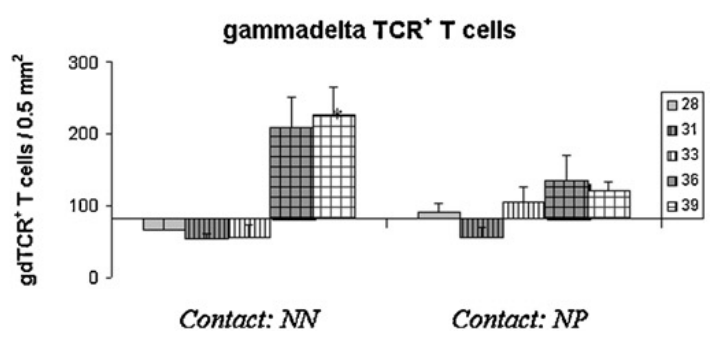

Fig. 2. The number of $\mathrm{CD}^{+}, \mathrm{CD}^{+}$and $\gamma \delta \mathrm{TCR}^{+}$cells in duodenum sections from inoculated and contact chickens of the NN group and the NP group compared to a baseline formed by chickens of the control group at 28,31 , 33,36 and 39 days of age. $\mathrm{CD}^{+}, \mathrm{CD}^{+}$and $\gamma \delta \mathrm{TCR}^{+} \mathrm{T}$ cells were stained and the amount of positive cells in an intestinal segment was counted. Mean of five chickens is shown. Baseline of the graphs is not set at zero, but at the mean amount of the control values. * Significant differences in

\subsection{Transmission between birds}

Transmission of the parasite was quantified for Groups 1-4 using the following measures:

- total number of infected contact birds per group;

- infection delay time per pair of birds and the total infection delay time per group.

The infection delay time, calculated per pair of birds, is defined as the number of days between the first day at which contact bird could have started excreting oocysts and the actual day oocysts were detected for the first time. The first day at which contact birds could have started shedding oocysts was two prepatent periods after inoculation (day 32), assuming sporulation time of oocysts to be negligible (Graat et al., 1994). The prepatent period for $E$. acervulina is approximately four days (Edgar, 1955; Joyner and Long, 1974; McDougald, 2003).

The day the contact bird actually started to excrete oocysts was based either solely on the McMaster oocyst counts (McM), or on combined McMaster and sedimentation-flotation test results $(\mathrm{McM} / \mathrm{SF})$. The sedimentation-flotation technique was more sensitive but did not allow quantification of output. When output started earlier according to the sedimentation-flotation test, this day was considered first day of excretion. Infection delay times were added for all pairs per group to obtain the total infection delay time.

\section{Results}

\subsection{Immunohistochemistry}

Numbers of T cells in birds at days $28,31,33,36$ or 39 are shown in Fig. 2. T cell counts did not differ significantly between non-primed $C_{N N}$ and primed $C_{N P}$ birds. $C_{N N}$ birds had significantly higher $\mathrm{CD} 4^{+} \mathrm{T}$ cell counts compared to the control birds on day 33. Non-significant increases of $\mathrm{CD}^{+} \mathrm{T}$ cells were observed in $I$ birds on days 31,33 , and $39\left(I_{N N}\right)$ and day $33\left(I_{N P}\right)$, and in $C_{N N}$ birds on day 39. $C_{N P}$ birds showed no marked increase in their intestinal $\mathrm{CD}^{+}$ $\mathrm{T}$ cell amount compared to control birds. The number of $\gamma \delta \mathrm{TCR}^{+} \mathrm{T}$ cells in $I_{N N}$ and $I_{N P}$ birds showed a steep increase at day 31 , and remained high until day $39\left(I_{N N}\right)$ and 36 $\left(I_{N P}\right)$. The number of $\gamma \delta \mathrm{TCR}^{+} \mathrm{T}$ cells of $C_{N N}$ birds increased at day 36 , whereas the primed $C_{N P}$ birds did not show a marked increase. Significant differences in $\mathrm{CD}^{+} \mathrm{T}$ cells and macrophages were not observed between inoculated and contact birds of the $N N$ and $N P$ groups when compared to control birds. The number of $\alpha \beta \mathrm{TCR}^{+} \mathrm{T}$ cells in control birds showed high fluctuations for all observed days (data not shown).

the numbers of the different $\mathrm{T}$ cell types between birds of the experimental group and control birds $(P<0.05)$. Note the difference in the scales. Plain columns represent measurements before transmission of the parasite to the contact bird could have taken place, striped columns represent data when transmission between inoculated and contact birds could have taken place and checked columns represent data when both birds are able to infect each other. 
Table 2

Oocyst output characteristics for $I$ and $C$ birds for all groups.

\begin{tabular}{|c|c|c|c|c|c|c|}
\hline Group & Bird & $\begin{array}{l}\text { \# of excreting birds } \\
(\mathrm{McM})^{\mathrm{a}}\end{array}$ & $\begin{array}{l}\text { \# of excreting birds } \\
(\mathrm{McM} / \mathrm{SF})^{\mathrm{a}}\end{array}$ & $\begin{array}{l}\text { OPG positive period } \\
\text { Mean \# of days (S.D.) }\end{array}$ & $\begin{array}{l}\text { Total oocyst output } \\
\text { Mean } \log _{10} \text { AUC (S.D.) }\end{array}$ & $\begin{array}{l}\text { Peak oocyst output } \\
\left.\text { Mean } \log _{10} \text { OPG (S.D. }\right)^{d}\end{array}$ \\
\hline $1: N N$ & $I_{N N}$ & 6 & 6 & $14.67(3.93) a$ & $6.29(0.30) a$ & $5.82(0.26) \mathrm{a}$ \\
\hline $1: N N$ & $C_{N N}$ & 6 & 6 & $13.83(0.98) a$ & $6.83(0.51) a$ & $6.57(0.53) a$ \\
\hline $2: N P$ & $I_{N P}$ & 6 & 6 & $13.00(3.10) a$ & $6.36(0.39) a$ & $6.03(0.51) a$ \\
\hline $2: N P$ & $C_{N P}$ & 5 & 6 & $6.00(5.76) b$ & $2.46(1.42) b$ & $2.18(1.21) b$ \\
\hline 3: $P N$ & $I_{P N}$ & 4 & 4 & $2.17(3.43) b$ & $1.59(1.40) \mathrm{b}$ & $1.63(1.44) \mathrm{bc}$ \\
\hline $3: P N$ & $C_{P N}$ & 6 & 6 & $14.50(2.17) a$ & $5.52(0.47) a$ & $5.23(0.44) a$ \\
\hline 4:PP & $I_{P P}$ & 1 & 5 & $1.00 b^{\mathrm{e}}$ & $0.49(1.20) c$ & $0.48(1.18) c$ \\
\hline 4: $P P$ & $C_{P P}$ & 1 & 1 & $1.00 \mathrm{~b}^{\mathrm{e}}$ & $0.24(0.58) c$ & $0.23(0.57) c$ \\
\hline
\end{tabular}

The mean of the OPG positive period, total oocyst output and peak oocyst output, followed by the standard deviation (S.D.) between brackets, is given per bird type for each group.

Values within the same column and with different letters $(a-c)$ differ significantly $(P<0.05)$.

a Number of birds excreting oocysts based solely on McMaster oocyst counts (McM) or on combined McMaster and sedimentation-flotation test results (McM/SF).

b Mean of the OPG positive period (in number of days) of (intermittent) oocyst excretion, which is the last day with OPG larger than zero minus the first day with OPG larger than zero.

c Mean of the $\log _{10}$-transformed AUC of the OPG, which represents total oocyst output during the entire oocyst excretion period.

d Mean of the highest $\log _{10}$-transformed OPG, which represents the peak of oocyst output.

e Only one $I_{P P}$ and one $C_{P P}$ bird were positive, therefore the standard deviation could not be calculated.

\subsection{Oocyst excretion patterns and transmission of infection}

After priming, the mean total oocyst excretion was $\log _{10} A U C=6.44$ (S.D. 0.26). The excretion data after inoculation or contact-infection are summarized in Table 2. Supplementary Tables 4-7 show oocyst counts and the associated infection delay times; in Table 3 the total infection delay time and the total number of infected contact birds are given per group.

All non-primed $I$ and $C$ birds $\left(I_{N N}, I_{N P}, C_{N N}, C_{P N}\right)$ excreted detectable numbers of oocysts (Table 2 and Supplementary Tables 4-6). Oocyst output in the primed $I_{P N}$ birds was detected in only four birds, after day 42 of the experiment (Supplementary Table 6), whereas all non-primed $C_{P N}$ birds excreted oocysts. Six primed $C_{N P}$ birds showed oocyst output (five birds positive according to McMaster counts and six according to sedimentation-flotation results) (Supplementary Table 5). Five primed $I_{P P}$ birds were observed to excrete oocysts (one according to McMaster counts and five birds according to sedimentation-flotation results), whereas the bird that did not show oocyst excretion according to both tests was the only one with its pen mate positive (Supplementary Table 7).

Non-primed $I$ and $C$ birds shed oocysts significantly longer, and had a higher $\log _{10}$ AUC and peak output compared to primed $I$ and $C$ birds (Table 2 ). The oocyst excretion parameters were significantly higher for $C_{P N}$ birds compared to $C_{N P}$ birds and were lowest for $C_{P P}$ birds. $I_{P N}$ birds had a significantly longer OPG positive period and higher $\log _{10}$ AUC than $I_{P P}$ birds (Table 2 ).

Oocyst output characteristics, e.g. period of excretion, total and peak output, were comparable between nonprimed $I$ and $C$ birds, irrespective of group (Table 2 ). Primed $I_{P N}$ and $C_{N P}$ birds showed comparable oocysts output characteristics and also primed $I_{P P}$ and $C_{P P}$ birds were not significantly different from each other regarding output data (Table 2).

In Fig. 3 mean oocyst excretion patterns, based on McMaster oocyst counts, are visualized for the four groups. Start of oocyst output in $C_{N P}$ birds was not delayed (day 33, Fig. 3B) compared to the $C_{N N}$ birds (Fig. $3 \mathrm{~A}$ ) but remained low for the duration of the experiment. On days 33-36 the $C_{N P}$ birds excreted a lower number of oocysts than the $I_{N P}$ birds, whereas these birds have experienced the same environment and infection history in numbers of oocysts $\left(50,000\right.$ in a single dose at inoculation on day 24 for $I_{N P}$ birds and the same dose at priming on day 6 for $C_{N P}$ birds). Oocysts were detected in faeces of $C_{N N}$ and $C_{P N}$ birds from day 33 onwards (Supplementary Tables 4 and 6). The peak of the latter was approximately six days later. In the PP group oocyst output was hardly detectable.

The infection delay times in the NP group were highly variable between pairs and were reduced when based on sedimentation-flotation results compared to McMaster counts (Supplementary Table 5). The lowest total infection delay time was found in the non-primed $N N$ group (10 days) and the highest total infection delay time and lowest num-

Table 3

Summary of transmission characteristics: total infection delay time and total number of infected contact birds per group.

\begin{tabular}{llll}
\hline \multirow{2}{*}{ Group } & \multicolumn{2}{l}{ Total infection delay time } & \multicolumn{2}{c}{ Total number infected $C$ birds } & \multicolumn{2}{c}{ McM } \\
\cline { 2 - 4 } & McM & McM/SF & 6 \\
$1: N N$ & 10 & 10 & 6 \\
$2: N P$ & 35 & 10 & 5 \\
$3: P N$ & 17 & 17 & 6 \\
$4: P P$ & 92 & 92 & 1 \\
\hline
\end{tabular}

\footnotetext{
a Sum of the infection delay times (based on Supplementary Tables 4-7) for all contact birds together per group.
} 

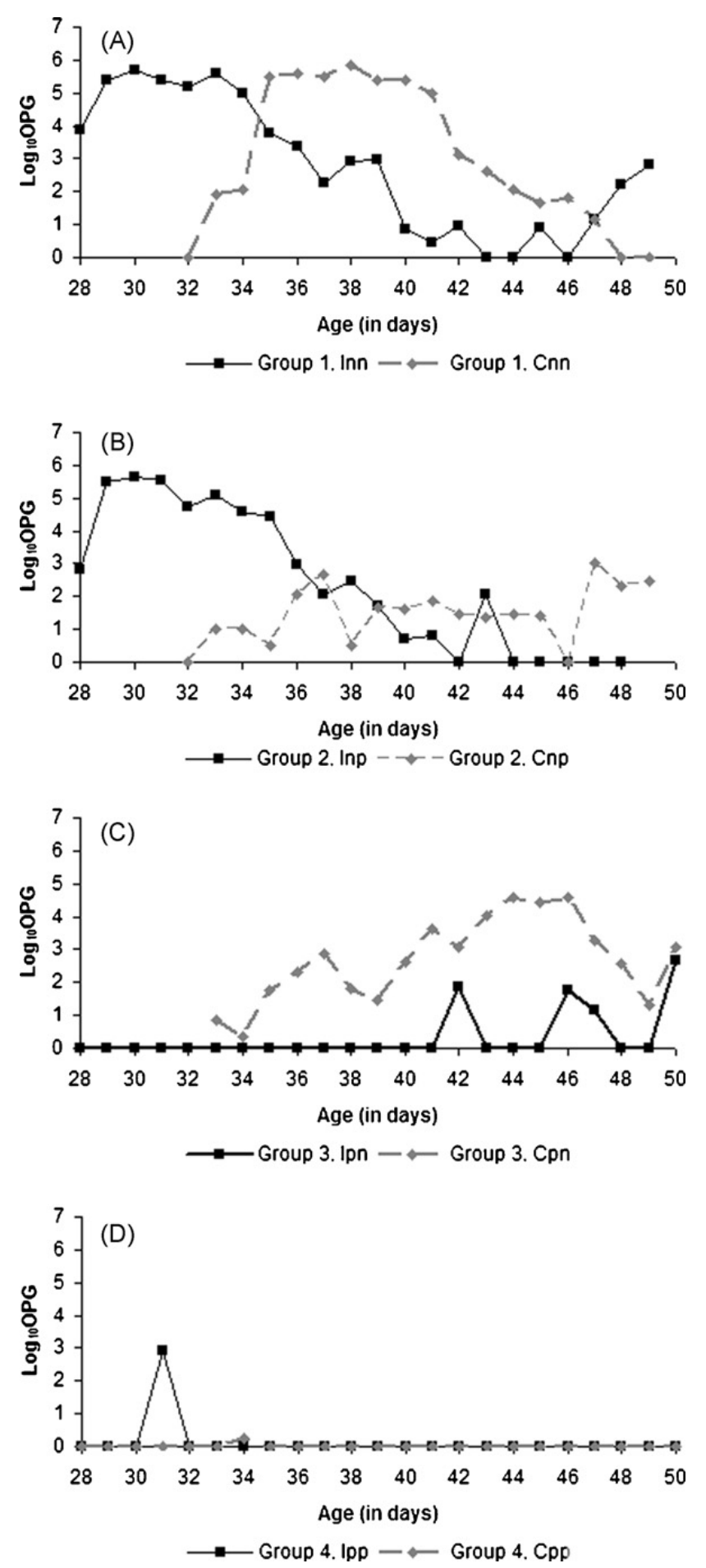

Fig. 3. Mean oocyst output for inoculated and contact birds $(n=6)$, based on McMaster oocyst counts. (A) Group $1(N N)$, (B) Group $2(N P),(C)$ Group $3(P N)$, (D) Group $4(P P)$.

ber of infected contact birds (one infected contact bird) was found in the primed PP group (Table 3).

\section{Discussion}

We studied the effect of heterogeneity in E. acervulina infection history of broilers on the acquired immunity in individual birds, and on the transmission characteristics of the parasite in the population.

\subsection{Effects of infection history on immune parameters}

None of the immunological cell types showed a significant sustained elevation level after infection in contact birds, and no single cell type could be linked to "protection level" or could predict oocyst output, infectivity, susceptibility or transmission between birds. In non-primed $C_{N N}$ birds, however, a significant increase in the number of $\mathrm{CD} 4^{+} \mathrm{T}$ cells was observed after contact-infection, but not in primed $C_{N P}$ chickens, despite exposure to similar amounts of oocysts. This suggests that $\mathrm{CD} 4^{+} \mathrm{T}$ cells do not take part in a protective immune reaction to infection of $E$. acervulina in primed chickens, but instead rise in number during a primary infection involving many parasites, a finding that is consistent with the study of Trout and Lillehoj (1996).

$\mathrm{CD}^{+} \mathrm{T}$ cells are known to be up-regulated after $E$. acervulina infections (Bessay et al., 1996; Swinkels et al., 2006), and are assumed to play a role in the recovery phase of infections (Lillehoj, 1988; Swinkels et al., 2007). The birds in our experiment, however, did not show a clear change in $\mathrm{CD}^{+} \mathrm{T}$ cell kinetics. This might be due to the gradual acquisition of parasites after contact-exposure, indicating that infections established via inoculation with a high dose may differ from "natural" contact-infection after exposure. The amount of oocysts ingested might have been too low to recruit a significant amount of $\mathrm{CD}^{+} \mathrm{T}$ cells.

In all chickens, except for the $C_{N P}$ birds an increase in $\gamma \delta \mathrm{TCR}^{+} \mathrm{T}$ cell response to an $E$. acervulina infection was observed from day 31 ( $I_{N N}$ and $I_{N P}$ birds) and day 36 ( $C_{N N}$ birds $)$ onwards, i.e. at the end of the endogenous phase of the life cycle in the intestinal tract (Fig. 2). Because $\gamma \delta \mathrm{TCR}^{+} \mathrm{T}$ cells are associated with development of intestinal epithelia (Boismenu and Havran, 1994) and may recognize damaged tissue (Schild et al., 1994), the increase is probably a reaction to intestinal damage after E. acervulina infections. In inoculated birds we see that the $\gamma \delta \mathrm{TCR}^{+} \mathrm{T}$ cell response remains high when the response in the non-primed contact chickens develops as well. This may indicate that the response to infection due to inoculation, the first infection cycle, is persistently high, but an alternative explanation may be that re-infection with excreted oocysts from the environment, a second infection cycle, is responsible. The latter explanation is very well possible, because oocyst excretion remains high during the second infection cycle, indicating considerable tissue damage and hence recruitment of $\gamma \delta \mathrm{TCR}^{+} \mathrm{T}$ cells (Fig. 2 and $3 \mathrm{~A}$ ). Primed $C_{N P}$ birds did not show an increase of $\gamma \delta \mathrm{TCR}^{+} \mathrm{T}$ cells. It is not clear whether this suggests that a primary response to infection results in different $\gamma \delta \mathrm{TCR}^{+} \mathrm{T}$ cell kinetics compared to a protective response after re-infection or that this finding can be solely explained by the reduced oocyst output and hence limited tissue damage in primed $C_{N P}$ birds.

Although we might have identified $\mathrm{T}$ cell markers for either a current $E$. acervulina infection $\left(\mathrm{CD} 4^{+} \mathrm{T}\right.$ cells) or intestinal damage due to an E. acervulina infection $\left(\gamma \delta \mathrm{TCR}^{+}\right.$ $\mathrm{T}$ cells), none of the single cell types showed a sustained elevated level after infection in primed or non-primed contact broilers. Therefore it can be concluded that none of the studied cell types correlates well with "protection level" nor was able to predict oocyst output, infectivity, suscepti- 
bility of previously infected birds or transmission between birds.

\subsection{Effects of primary infection on oocyst output and transmission between birds}

It is known that after ingestion of oocysts, the induced immune response can reduce oocyst output after reinfection (Lillehoj, 1988; Williams, 1995; Blake et al., 2005). In this experiment, the infection history reduced oocyst output in inoculated birds and contact-exposed birds. The non-primed $C_{P N}$ birds showed a delayed peak of excretion compared to the non-primed $C_{N N}$ birds, suggesting a delayed transmission of Eimeria. Although oocyst output by the primed $I_{P N}$ birds was hardly detectable, these birds were apparently sufficiently infectious to establish contact-infection, indicating the usefulness of transmission experiments, which mimics natural infections better than experiments with "artificially" inoculated birds only. Oocyst output by the primed $C_{N P}$ birds was significantly lower compared to that of the non-primed $C_{N N}$ and $C_{P N}$ birds, indicating a reduced susceptibility. However, transmission of infection did occur, which suggests that the acquired immunity was not sufficient to resist the high number of oocysts excreted by the $I_{N P}$ bird. Whether or not this was the case for the $P P$ combination cannot be determined, as it cannot be determined whether the apparent absence of oocysts in the faeces of $C_{P P}$ birds is due to the fact that the birds were not infected or that the number was below the detection limit of the tests. Limitations of detection are demonstrated by the two $C_{P N}$ birds and one $C_{P P}$ bird that excreted oocysts, whereas their inoculated pen mates did not show oocyst output for the entire duration of the experiment. This suggests that small numbers of oocysts remain undetectable for both techniques. A quantitative PCR technique might be more appropriate to improve the detection limit for faecal samples with small numbers of oocysts (Blake et al., 2008; Morgan et al., 2009; Velkers et al., 2010).

Non-primed inoculated and contact birds do not differ in infection history before day 24 (inoculation), but do differ in timing and dose of ingestion of oocysts after that. Inoculated and contact birds most likely repeatedly ingested variable numbers of oocysts from the environment after day 28 , but inoculated birds also received a single inoculation dose of 50,000 oocysts at day 24 . Because duration of output and total output were comparable between non-primed inoculated and contact birds, the inoculation was apparently irrelevant. However, a single inoculation 18 days earlier (priming) did change the excretion pattern: priming itself caused a single high peak, and after day 24 total output and duration were significantly lower and shorter than in non-primed birds. Apparently, time intervals between oocyst ingestion are sometimes crucial for subsequent oocyst output (primed birds), and in some cases not at all (non-primed $I$ and $C$ birds). Only transmission experiments or experiments in which birds are allowed to re-infect themselves automatically provide a realistic timing of ingestions. It shows that simple challenge experiments, using high doses of oocysts, may give different results, and that oocyst output cannot be simply translated to predictions on outcome of infection or spread of the disease.

Another observation on timing of oocyst ingestions can be made by comparing the second infection cycles of nonprimed inoculated birds $\left(I_{N N}\right.$ and $\left.I_{N P}\right)$, and of primed $C_{N P}$ birds. These infection cycles correspond with the excretion around days $32-36$ and the $\gamma \delta \mathrm{TCR}^{+} \mathrm{T}$ cell responses at days 36 and 39. Before the second infection cycle all these birds had experienced the same infection history with respect to numbers of oocysts $(50,000$ in a single dose), but they differed in the moment in life these oocysts had been given. Because the inoculated birds showed much higher oocyst excretion and elevated $\gamma \delta \mathrm{TCR}^{+} \mathrm{T}$ cell levels than the primed contact birds, this suggests that inoculation at day 24 of $I_{N N}$ and $I_{N P}$ birds resulted in a less effective protective immune response than the priming inoculation at day 6 of $C_{N P}$ birds. Apparently, there is a delay in the immune response, which may have considerable impact on dynamics in a larger population, and which is not yet covered by present models described by Klinkenberg and Heesterbeek (2007) and Severins et al. (2007).

In conclusion, contrary to our expectation that the measured immune parameters could indicate acquired immunity by predicting level of protection against oocyst output and transmission, no cell types were identified in this study to confirm this assumption. However, it was shown that primary infection could significantly reduce oocyst output after secondary infection. Furthermore, the natural infection of contact birds by exposure to inoculated birds has shown that undetectably low oocyst outputs can cause contact-infections, where the level of the subsequent oocyst output in contact birds depends on the level of acquired immunity obtained through the infection history of these birds. The combined study of withinhost and between-host dynamics also has revealed that a delay between infection and development of immunity can occur, which can have great impact on infection dynamics in flocks.

\section{Acknowledgement}

We would like to thank Hans Vernooij for his valuable help and advice during the statistical analysis.

\section{Appendix A. Supplementary data}

Supplementary data associated with this article can be found, in the online version, at doi:10.1016/j.vetpar.2010.07.005.

\section{References}

Bessay, M., Le Vern, Y., Kerboeuf, D., Yvore, P., Quere, P., 1996. Changes in intestinal intra-epithelial and systemic T-cell subpopulations after an Eimeria infection in chickens: comparative study between $E$. acervulina and E. tenella. Vet. Res. 27, 503-514.

Blake, D.P., Hesketh, P., Archer, A., Carroll, F., Shirley, M.W., Smith, A.L., 2005. The influence of immunizing dose size and schedule on immunity to subsequent challenge with antigenically distinct strains of Eimeria maxima. Avian Pathol. 34, 489-494.

Blake, D.P., Qin, Z., Cai, J., Smith, A.L., 2008. Development and validation of real-time polymerase chain reaction assays specific to four species of Eimeria. Avian Pathol. 37, 89-94. 
Boismenu, R., Havran, W.L., 1994. Modulation of epithelial cell growth by intraepithelial gamma delta T cells. Science 266, 1253-1255.

Chapman, H.D., 1997. Biochemical, genetic and applied aspects of drug resistance in Eimeria parasites of the fowl. Avian Pathol. 26, 221-244.

Claerebout, E., Vercruysse, J., 2000. The immune response and the evaluation of acquired immunity against gastrointestinal nematodes in cattle: a review. Parasitology 120, 25-42.

Cornelissen, J.B.W.J., Swinkels, W.J.M., Boersma, W.A., Rebel, J.M.J., 2009. Host response to simultaneous infections with Eimeria acervulina, maxima and tenella: a cumulation of single responses. Vet. Parasitol. 162, 58-66.

Edgar, S.A., 1955. Sporulation of oocysts at specific temperatures and notes on the prepatent period of several species of avian coccidia. J. Parasitol. 41, 214-216.

Graat, E.A.M., Henken, A.M., Ploeger, H.W., Noordhuizen, J.P.T.M., Vertommen, M.H., 1994. Rate and course of sporulation of oocysts of Eimeria acervulina under different environmental conditions. Parasitology 108 (Pt 5), 497-502.

Graat, E.A.M., Ploeger, H.W., Henken, A.M., De Vries Reilingh, G., Noordhuizen, J.P.T.M., Van Beek, P.N.G.M., 1996. Effects of initial litter contamination level with Eimeria acervulina on population dynamics and production characteristics in broilers. Vet. Parasitol. 65, 223-232.

Graat, E.A.M., van der Kooij, E., Frankena, K., Henken, A.M., Smeets, J.F.M., Hekerman, M.T.J., 1998. Quantifying risk factors of coccidiosis in broilers using on-farm data based on a veterinary practice. Prev. Vet. Med. 33, 297-308.

Joyner, L.P., Long, P.L., 1974. The specific characters of the Eimeria, with special reference to the coccidia of the fowl. Avian Pathol. 3, 145-157.

Klinkenberg, D., Heesterbeek, J.A.P., 2007. A model for the dynamics of a protozoan parasite within and between successive host populations. Parasitology 134, 949-958.

Lillehoj, H.S., 1988. Influence of inoculation dose, inoculation schedule, chicken age, and host genetics on disease susceptibility and development of resistance to Eimeria tenella infection. Avian Dis. 32, 437-444.

Long, P.L., Rowell, J.G., 1958. Counting oocysts of chicken coccidia. Lab. Pract. 7, 515-518, 534

Long, P.L., Millard, B.J., Joyner, L.P., Norton, C.C., 1976. A guide to laboratory techniques used in the study and diagnosis of avian coccidiosis. Folia Vet. Lat. 6, 201-217.

McDougald, L.R., 2003. Protozoal infections. In: Saif, Y., Barnes, H.J., Glisson, J.R., Fadly, A.M., McDougald, L.R., Swayne, D.E. (Eds.), Diseases of Poultry. Iowa State Press, Ames, Iowa, pp. 973-1022.

Mes, T.H.M., 2003. Technical variability and required sample size of helminth egg isolation procedures. Vet. Parasitol. 115, 311-320.

Morgan, J.A.T., Morris, G.M., Wlodek, B.M., Byrnes, R., Jenner, M., Constantinoiu, C.C., Anderson, G.R., Lew-Tabor, A.E., Molloy, J.B., Gasser, R.B., Jorgensen, W.K., 2009. Real-time polymerase chain reaction (PCR) assays for the specific detection and quantification of seven Eimeria species that cause coccidiosis in chickens. Mol. Cell. Probes 23, 83-89.

Peek, H.W Landman, W.J.M. 2003. Resistance to anticoccidial drugs of Dutch avian Eimeria spp. field isolates originating from 1996, 1999 and 2001. Avian Pathol. 32 (4), 391-401.

Roberts, M.G., Heesterbeek, J.A.P., 1995. The Dynamics of Nematode Infections of Farmed Ruminants. Parasitology 110, 493-502.

Roberts, M.G., Heesterbeek, J.A.P., 1998. A simple parasite model with complicated dynamics. J. Math. Biol. 37, 272-290.

Rose, M.E., 1987. Immunity to Eimeria infections. Vet. Immunol. Immunopathol. 17, 333-343.

Schild, H., Mavaddat, N., Litzenberger, C., Ehrich, E.W., Davis, M.M., Bluestone, J.A., Matis, L., Draper, R.K., Chien, Y.H., 1994. The nature of major histocompatibility complex recognition by gamma delta T cells. Cell 76, 29-37.

Severins, M., Klinkenberg, D., Heesterbeek, H., 2007. Effects of heterogeneity in infection-exposure history and immunity on the dynamics of a protozoan parasite. J. R. Soc. Interface 4, 841-849.

Shirley, M.W., Smith, A.L., Tomley, F.M., 2005. The biology of avian Eimeria with an emphasis on their control by vaccination. Adv. Parasitol. 60, 285-330.

Stiff, M.I., Bafundo, K.W., 1993. Development of immunity in broilers continuously exposed to Eimeria sp. Avian Dis. 37, 295-301.

Swinkels, W.J.C., Post, J., Cornelissen, J.B., Engel, B., Boersma, W.J.A., Rebel, J.M.J., 2006. Immune responses in Eimeria acervulina infected oneday-old broilers compared to amount of Eimeria in the duodenum, measured by real-time PCR. Vet. Parasitol. 138, 223-233.

Swinkels, W.J.C., Post, J., Cornelissen, J.B., Engel, B., Boersma, W.J.A., Rebel, J.M.J., 2007. Immune responses to an Eimeria acervulina infection in different broilers lines. Vet. Immunol. Immunopathol. 117, 26-34.

Trout, J.M., Lillehoj, H.S., 1996. T lymphocyte roles during Eimeria acervulina and Eimeria tenella infections. Vet. Immunol. Immunopathol. 53, 163-172.

Velkers, F.C., Blake, D.P., Graat, E.A.M., Vernooij, J.C.M., Bouma, A., de Jong M.C.M., Stegeman, J.A., 2010. Quantification of Eimeria acervulina in faeces of broilers: comparison of McMaster oocyst counts from $24 \mathrm{~h}$ faecal collections and single droppings to real-time PCR from cloacal swabs. Vet. Parasitol. 169, 1-7.

Velthuis, A.G.J., Bouma, A., Katsma, W.E.A., Nodelijk, G., De Jong, M.C.M. 2007. Design and analysis of small-scale transmission experiments with animals. Epidemiol. Infect. 135, 202-217.

Williams, R.B., 1995. Epidemiological studies of coccidiosis in the domesticated fowl (Gallus gallus). IV: Reciprocity between the immune status of floor-reared chickens and their excretion of oocysts. Appl. Parasitol. 36, 290-298.

Williams, R.B., 1998. Epidemiological aspects of the use of live anticoccidial vaccines for chickens. Int. J. Parasitol. 28, 1089-1098. 